\title{
Actualización: Factores psicosociales y su asociación con la enfermedad cardiovascular
}

Psychosocial factors and its association with cardiovascular disease

Sergio Terrasa *

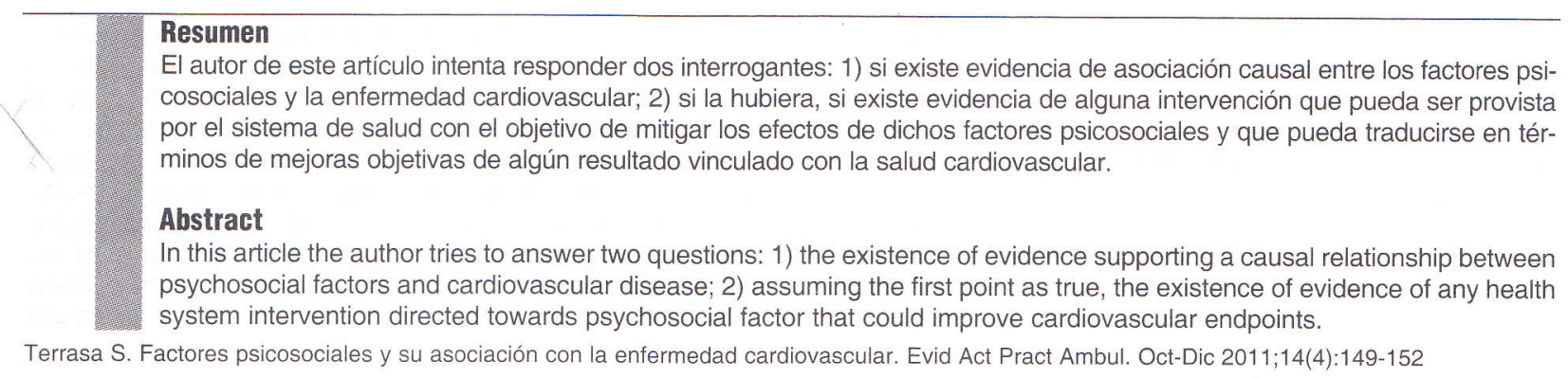

Conceptualizaciones generales respecto de los factores psicosociales y su asociación con resultados en salud

Entre los factores psicosociales que pueden asociarse a resultados adversos en salud (y particularmente a un aumento de la incidencia de eventos cardiovasculares) Rozanski y col. ${ }^{1}$, distinguen dos grandes categorías:

a) una relacionada con el individuo (o intrínseca) y que ha sido descripta como la de los factores emocionales y que incluye a los trastornos afectivos (ansiedad/depresión), a la hostilidad, y a la tendencia a la ira;

b) otra asociada al ambiente (o extrínseca), descripta como la de los estresores crónicos y que incluye a la falta de soporte social, al bajo estatus socioeconómico, al estrés laboral cróni$\mathrm{co}$, al estrés de la pareja y al estrés del cuidador.

\section{Factores emocionales}

Entre los factores emocionales, el más estudiado ha sido la depresión. Como ejemplo de asociación documentada, citamos la de Lesperance y col. ${ }^{2}$, que observó un claro gradiente (efecto tipodosis respuesta) entre la magnitud de los síntomas depresivos (valorados mediante el puntaje de Beck, que asigna probabilidades crecientes de depresión a mayor puntaje) y la incidencia de nuevos eventos cardiovasculares (CV) en pacientes que ya habían sufrido un infarto agudo de miocardio (IAM) y fueron seguidos durante cinco años. Esta asociación se hizo evidente aún ante bajos niveles de dichos síntomas depresivos. Ver figura 1.

Figura 1: asociación entre la magnitud de los síntomas depresivos valorados a través de puntaje de Beck y la mortalidad cardiovascular a cinco años en pacientes que ya habían sufrido un infarto agudo de miocardio.

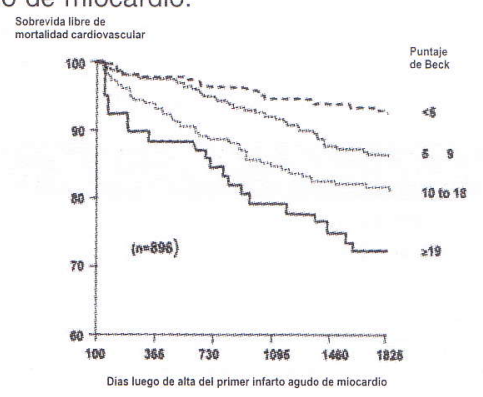

Fuente: Lesperance $\mathrm{F}$ y col. Five-year risk of cardiac mortality in relation to initia severity and one-year changes in depression symptoms after myocardial infarction. Circulation 2002;105:1049-53.
En 2007, Van del Koy y col. ${ }^{3}$ publicaron una revisión sistemática que reportó una asociación entre la presencia de depresión y un aumento en el riesgo de IAM (OR* 1,60; IC95\% 1,34 a 1,92); mientras que Pan y col. ${ }^{4}$ publicaron otra en 2011 que documentó la asociación entre depresión y el riesgo de accidente cerebrovascular (ACV) (HR* 1,45 IC95\% 1,29 a 1,63) y riesgo de ACV fatal (HR 1,55 IC95\% 1,25 a 1,93) luego de meta-analizar los resultados ajustados por potenciales confundidores de 28 cohortes que habían incluido 317.540 participantes que habían sido seguidos durante un períodos de dos a 29 años.

Respecto de la tendencia a la ira y a la hostilidad, la evidencia es mucho más débil pero coincidente ${ }^{1}$, dado que ha sido documentada la asociación entre estas características de la personalidad y algunos marcadores subclínicos del avance del proceso de aterosclerosis (valoraciones sucesivas en un mismo paciente de su anatomía coronaria o de las carótidas, etc.).

\section{Estresores crónicos}

\section{Soporte social}

Respecto del soporte social, también Rozanski y col. ' describen dos grandes conceptos relacionados al mismo:

a) La forma y/o estructura de las redes sociales que tiene un individuo y la frecuencia de contacto que tiene éste con dicha red. b) El soporte funcional, que a su vez puede ser dividido conceptualmente en soporte funcional recibido (definido "objetivamente" como el tipo y la cantidad de recursos presentes en dicha red) y soporte funcional percibido (con una valoración más subjetiva y relacionado con la satisfacción del individuo respecto de dicho soporte y la percepción de que los recursos estarán realmente presentes cuando él los necesite). Además describen varios aspectos del soporte funcional: instrumentals, financiero, informacional**, evaluativo ${ }^{\S \S}$ y emocional.

Respecto de la evidencia que sustenta la asociación entre el soporte provisto por las relaciones sociales y la del riesgo de mortalidad, vale destacar la revisión sistemática publicada por Holt-Lunstad $\mathrm{J}$ y col. ${ }^{5}$ (resumida y comentada en un número anterior de EVIDENCIA ). Estos autores evaluaron 148 publicaciones originales que habían incluido 308.849 casos y establecieron dos grandes categorías de soporte social:

1) La de los aspectos funcionales: a) de apoyo recibido (emocional, de información o material); b) de percepción de apoyo;

sun ejemplo de soporte funcional instrumental es que un individuo con alguna discapacidad pueda ser ayudado por alguien de su red en alguna de sus tareas domésticas. ** Un ejemplo de soporte funcional informacional es el de tener ayuda para enterarse de que existe la posibilidad de tramitar un certificado de discapacidad que permitirá acceder a algunos beneficios como la obtención de medicación en forma gratuita.

ss Un ejemplo de soporte funcional evaluativo es contar con ayuda para valorar que existe una situación de alto riesgo de caídas en el hogar y decidir que colocar un pasamanos en una escalera sería una buena estrategia para prevenirlas.

* Servicio de Medicina Familiar y Comunitaria del Hospital Italiano de Buenos Aires (HIBA) y Departamento de Salud Pública del Instituto Universitario HIBA. sergio.terrasa@hiba.org.ar 
c) de soledad (sentimientos de aislamiento, de apatía o de no pertenencia).

2) La de los aspectos estructurales: a) de situación de pareja, b) de redes sociales (ej. número de contactos en el mundo real); c) de integración (grado de participación en diversas relaciones y/o actividades, sentido de comunidad e identificación con algún rol social); d) de convivencia (vivir sólo vs. con otro); e) de aislamiento social (falta generalizada de relaciones sociales y de comunicación, de participación y/o ausencia de confidentes); f) medidas complejas de integración con varios componentes de los anteriores.

Estos autores documentaron que los individuos con adecuadas relaciones sociales tuvieron una probabilidad de supervivencia un $50 \%$ mayor $(\mathrm{OR}=1,50$; $1 \mathrm{C} 95 \%$ 1,42 a 1,59) que aquellos cuyas relaciones sociales fueron deficientes o insuficientes, $y$ que se mantuvo constante, luego de ajustar por posibles factores de confusión como la edad, el sexo y el diagnóstico clínico. Sin bien las diferencias fueron significativas $(p<0,001)$ para todos los aspectos de las relaciones sociales considerados, la asociación con supervivencia fue más fuerte con las medidas complejas de integración $(\mathrm{OR}=1,91$; IC95\% 1,63 a 2,23) y menor con los indicadores binarios (OR $=1,19$, IC $95 \%$ : 0,99 a 1,44).

\section{Estrés laboral}

Tomando como guía a la revisión de Fernández López y col.7, podemos decir que existen dos principales modelos teóricos que han intentado conceptualizar la asociación entre el estrés laboral y resultados adversos en salud:

a) El modelo desarrollado por Karasell y Theorell denominado de "Demanda/control sobre el estrés laboral" propone que el estrés laboral no sólo depende de las demandas laborales que sufre el empleado sino también del control, la creatividad y la posibilidad que este tiene de implementar decisiones propias sobre la tarea que está haciendo. A mayor demanda laboral y menor autonomía para realizar sus tareas", mayor estrés laboral. Ver figura 2.

Figura 2: modelo propuesto por Karasell y Theorell para conceptualizar el estrés laboral, denominado de "Demanda/control sobre el estrés laboral".

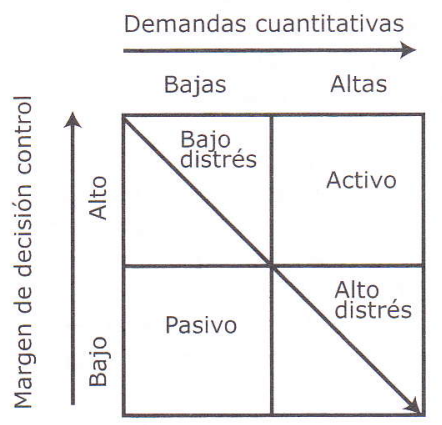

Modificado de: Fernández Lopez y col. El estrés laboral: un nuevo factor de riesgo. ¿Qué sabemos y qué podemos hacer? Aten Primaria 2003;31(8):000-0.

b) El modelo desarrollado por Siegrist y denominado "De crisis de gratificación en el trabajo", propone que el estrés laboral resulta del juego de las tensiones entre las situaciones dependientes del ambiente laboral (extrínsecas) y las que pone en juego cada individuo (intrísecas). Entre las dependientes del entorno laboral figuran las relacionadas con las exigencias del mismo y las vinculadas con la recompensa a las demandas y obligaciones (ingresos, seguridad y/o estabilidad laboral, reconocimiento social que ese trabajador obtiene por su rol, posibilidades de promoción laboral, etc.); y entre las del propio trabajador, podemos mencionar a como repercute dicha situación en su autoestima, cuanto se sobre-involucra en sus tareas laborales, etc.

Figura 3: modelo propuesto por Siegrist para conceptualizar el estrés laboral denominado "De crisis de gratificación en el trabajo".

\begin{tabular}{|c|c|c|}
\hline \multicolumn{3}{|c|}{$\begin{array}{l}\text { Implicación excesiva en el trabajo } \\
\text { (overcommirment) }\end{array}$} \\
\hline $\begin{array}{l}\text { Intrínseco } \\
\text { (personal) }\end{array}$ & $\begin{array}{l}\text { Alto } \\
\text { esfuerzo }\end{array}$ & $\begin{array}{l}\text { Baja } \\
\text { recompensá }\end{array}$ \\
\hline $\begin{array}{l}\text { Extrínseco } \\
\text { (situación) }\end{array}$ & $\begin{array}{l}\text {-Demandas } \\
\text {-Obligaciones }\end{array}$ & $\begin{array}{l}\text {-Ingresos } \\
\text {-Estima } \\
\text {-Seguridad/promoción } \\
\text { laboral }\end{array}$ \\
\hline
\end{tabular}

Modificado de: Fernández Lopez y col. El estrés laboral: un nuevo factor de riesgo. ¿Qué sabemos y qué podemos hacer? Aten Primaria 2003;31(8):000-0

Estos dos modelos fueron puestos a prueba en forma prospectiva por Kivimäki M y col. ${ }^{8}$ a través de un estudio de cohorte finlandesa de 812 empleados metalúrgicos que fueron seguidos desde 1973 hasta $2001^{\$ \varsigma \S}$, y cuya publicación también fue resumida en EVIDENCIA ${ }^{9}$. Luego del ajuste por edad y sexo, los empleados con elevado estrés laboral entendido como alta demanda con poco control sobre la tarea tuvieron más del doble de riesgo de muerte CV que los de bajo estrés laboral, ocurriendo lo mismo con la valoración del balance entre el esfuerzo y la recompensa (ver tabla 1) y permaneciendo esta asociación luego de ajustar por potenciales factores de confusión como el tipo de trabajo, variables biológicas (niveles de tensión arterial, colesterol, tabaquismo, etc.) y por el autoreporte de sus hábitos de vida.

Tabla 1: asociación entre el grado de estrés laboral y la mortalidad cardiovascular en una cohorte de empleados metalúrgicos de Finlandia seguidos entre 1973 y 2001

\begin{tabular}{l|c|c}
\multicolumn{1}{c|}{ Grado de estrés laboral } & RR (IG95\%) \\
\hline $\begin{array}{l}\text { Entendido como alta demanda } \\
\text { laboral con poco control sobre la } \\
\text { tarea }\end{array}$ & Bajo & 1 \\
\cline { 2 - 3 } $\begin{array}{l}\text { Entendido como disparidad entre el } \\
\text { esfuerzo y la recompensa }\end{array}$ & Blto & $2,2(1,16$ a 4,17) \\
\cline { 2 - 3 } & Alto & $2,36(1,26$ a 4.42) \\
\hline
\end{tabular}

aBajo salario, poca aprobación social y bajas oportunidades de progreso.

\section{Aspectos negativos de los vínculos cercanos}

La repercusión de los vínculos cercanos en la incidencia de

"Un ejemplo de bajo control y/o decisión sobre la tarea es el de estar todo el tiempo en el mismo puesto de trabajo de una línea de producción de una pieza mecánica. S5s Los datos de estrés laboral se obtuvieron de auto cuestionarios que evaluaron ambas dimensiones y los datos de mortalidad cardiovascular se obtuvieron del registro nacional de mortalidad. El seguimiento promedio fue de 25,6 años. 
enfermedad coronaria (EC) fue evaluada por De Vogli y col. ${ }^{10} \mathrm{a}$ través de una cohorte londinense que siguió durante 12 años a 8.499 personas. Luego de ajustar por potenciales factores de confusión como edad, sexo, estado civil, nivel de empleo, obesidad, hipertensión arterial, diabetes mellitus, nivel de colesterol, apoyo social y recursos de apoyo, se observó una asociación con efecto dosis-respuesta entre las relaciones cercanas altamente estresantes (el 70\% correspondieron a relaciones de pareja) y la incidencia de EC (HR 1,34; IC95\% 1,10 a $1,63^{\star * * *}$ )

\section{Estrés del cuidador}

La cohorte de las enfermeras informó una duplicación del riesgo cardiovascular a cuatro años en quienes tenían el esposo enfermo ${ }^{11}$; mientras que la investigación publicada por Freedman y col. ${ }^{12}$ reportó que ser cuidador se asoció a menor mortalidad que no ser cuidador. Ante los resultados descriptos previamente vale destacar que Schultz y col. ${ }^{13}$ sólo encontraron evidencia de peores resultados en salud solo cuando el cuidador siente a este rol como una carga y asociado a la presencia de sentimientos negativos.

\section{Estudios que evaluaron variables combinadas}

No podemos dejar de lado en esta revisión, el estudio de casos y controles publicado por Yussuf y col y conocido con el nombre INTERHEART ${ }^{14}$, que incluyó 12.461 casos y 14.637 controles de 52 países de todos los continentes y que avalaría la universalidad de los factores de riesgo cardiovascular (1.237 de los casos provinieron de Sudamérica).

Además de incluir en el análisis a la edad y el sexo, a información sobre el estilo de vida (consumo de alcohol y tabaco, práctica de ejercicio y consumo de vegetales), a la obesidad, a los valores de presión arterial, de lípidos y diabetes, los autores de esta investigación incluyeron una variable llamada "indice psicosocial" (PS) que trató de sintetizar información sobre el ánimo (depresión), la falta de control, el estrés percibido en la casa y/o en el trabajo, el estrés financiero y las experiencias vitales adversas. La variable "índice psicosocial" se asoció en forma directa a la ocurrencia de IAM, tanto en mujeres (OR 3,49 ; IC95\% 2,41 a 5,04) como en varones (OR 2,58; 2,11 a 3,14 ) con un riesgo poblacional atribuible* respectivamente de $40 \%(28,6$ a 52,6$)$ y $25,3 \%(18,2$ a 34$)$

\section{¿Qué podemos ofrecer desde el sistema de salud?}

Luego de lo resumido previamente, consideramos que existe sobrada evidencia sobre la asociación independiente entre los factores psicosociales y la morbi-mortalidad CV.

El segundo interrogante que compete a esta revisión es si existe alguna intervención que implementada desde el sistema de salud haya demostrado mejorar algún resultado en salud.

En este sentido vale destacar el ensayo clínico recientemente publicado por Gulliksson y col. ${ }^{15 t+\dagger}$, que tomó lugar entre 1996 y 2002, en Upsala (Suecia) y en el que 362 pacientes que habían sufrido un IAM y/o recibido angioplastia o cirugía de revascularización coronaria fueron aleatorizados a dos grupos: a) grupo control de cuidados usuales; b) grupo intervención que consistió en cuidados usuales más 20 sesiones grupales de terapia cognitivo conductual coordinadas por enfermeros y psicólogos especializados, que tenían como objetivo ayudarlos a desarrollar herramientas para el manejo del estrés. En el dispositivo de la rama "intervención" se trabajó sobre cinco ejes: 1) educativo (información sobre el aparato $\mathrm{CV}$, sobre los estilos de vida saludable, sobre la relación entre el estrés y la enfermedad CV, etc.); 2) de automonitoreo de signos de estrés con promoción de las habilidades para que el paciente saque conclusiones sobre él mismo; 3) de entrenamiento en habilidades para aprender como responder constructivamente en vez de "reaccionar" frente al estrés; 4) de reestructuración cognitiva; 5) de desarrollo espiritual (discusión sobre valores, qué significa una buena calidad de vida, etc.).

Luego de ocho años de seguimiento, estos autores documentaron una reducción estadísticamente significativa en la recurrencia de enfermedad CV y de IAM en el grupo asignado a terapia cognitivo conductual (NNT 9 a 10).

Más allá de los resultados alentadores de la investigación que acabamos de resumir, no podemos dejar de mencionar los resultados de la revisión sistemática de Whalley y col. ${ }^{16}$ que reportó que existe poca evidencia de que las intervenciones psicosociales en pacientes con enfermedad coronaria se asocien a una menor mortalidad, a menor probabilidad de revascularización ni a menor incidencia de IAM no fatales; reportando sólo una modesta tendencia de reducción de la mortalidad cardíaca (RR 0,80; IC95\% 0,64 a 1,00) ${ }^{\dagger+t+}$.

\section{Conclusión}

Respecto de la primera de las preguntas que motivaron esta revisión y basándonos en los resultados descriptos previamente, podemos decir que existe fuerte evidencia respecto de la asociación entre variados factores psicosociales y algunos resultados adversos en salud CV. Aprovechamos para ofrecer al lector una adaptación del modelo teórico de Rozanski y col. ${ }^{1}$ que se describe en la figura 4 y que intenta esquematizar algún tipo de vinculación causal.

Figura 4: modelo teórico de Rozanski que intenta explicar la asociación entre los factores psicosociales y la mayor incidencia de enfermedad cardiovascular.

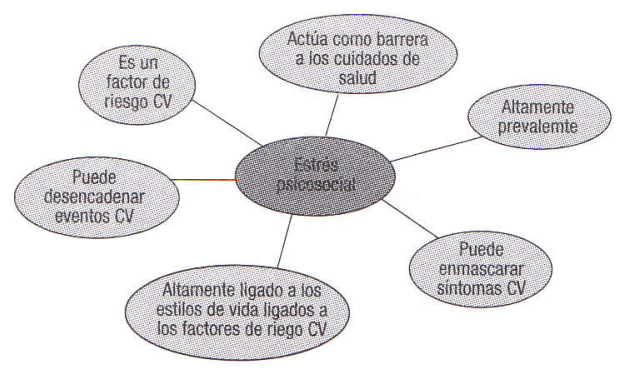

CV: cardiovascular. Modificado de: Rozanski A y col. The Epidemiology, Pathophysiology, and Management of Psychosocial Risk Factors in Cardiac Practice. J Am Coll Cardiol 2005:45:637-51.

Respecto de la segunda pregunta, la respuesta es menos clara por ahora y quizás siempre lo sea. Consideramos que seguramente irá surgiendo evidencia respecto de la efectividad de

\footnotetext{
Vale destacar que luego realizar un ajuste por la presencia de problemas afectivos y depresión, la relación se atenuó pero siguió siendo estadísticamente significativa (HR 1 25; C95\% 1,02 a 1,55

"tt Sepa el lector que fue este trabajo de Gulliksson y col. el que nos motivó a varias personas de nuestra institución a realizar esta revisión del tema.

titt Estos autores sólo encontraron que este tipo de intervenciones se asoció a resultados intermedios como mejorías en los puntajes de depresión con una diferencia media estandarizada (DME) de $-0,21$ (IC95\% - -0,35 a -0,08) y de ansiedad DME - $-0,25(-0,48$ a $-0,03)$; y con mejores resultados en pacientes con personalidad de tipo A $(p=0,03$ )
} 
algunas intervenciones pasibles de ser implementadas desde el sistema de salud para ayudar a nuestros pacientes y familias a mitigar los efectos dañinos del estrés psicosocial sobre su salud.

Sin embargo, no tenemos mucha esperanza de que puedan documentarse la efectividad de intervenciones capaces de operar al margen de las causas sistémicas de sufrimiento cróni- co (al que en esta revisión llamamos estrés psicosocial). Estas causas sistémicas de sufrimiento crónico tienen un fuerte componente familiar, social, laboral, económico y político; jugando un rol clave en ellas la pobreza, la inequidad, la explotación, el maltrato, la exclusión y la marginalidad. Por lo tanto, dependen de soluciones políticas, en el sentido amplio de la palabra.

*Ver glosario.

Recibido el 01/11/2011 y aceptado el 01/12/2011

\section{Referencias}

1. Rozanski A y col. The Epidemiology, Pathophysiology, and Management of Psychosocial Risk Factors in Cardiac Practice. J Am Coll Cardiol 2005;45:637-51.

2. Lesperance $F$ y col. Five-year risk of cardiac mortality in relation to initial severity and one-year changes in depression symptoms after myocardial infarction. Circulation 2002;105:1049-53.

3. Van del Koy Ky col. Depression and the risk for cardiovascular diseases: systematic review and meta analysis. Int J Geriatr Psychiatry. 2007 Jul;22(7):613-26.

4. Pan A y col. Depression and Risk of Stroke Morbidity and Mortality A Meta-analysis and Systematic Review. 2011;306(11):1241-1249. doi:10.1001/jama.2011.1282.

5. Holt-Lunstad J y col (2010) Social Relationships and Mortality Risk: A Meta-analytic Review. PLoS Med 7(7): e1000316. doi:10.1371/joumal.pmed.1000316

6. Vilosio J. Las relaciones sociales constituyen un factor de riesgo para la salud. Evid Act Pract Ambul 14(2) 49. Abr-Jun 2011. Comentado de: Holt-Lunstad J y col (2010) Social Relationships and Mortality Risk: A Meta-analytic Review. PLoS Med 7(7): e1000316. Disponible resumido en castellano en: http://www.foroaps.org/files/rel\%20socia.pdf

7. Fernández Lopez y col. El estrés laboral: un nuevo factor de riesgo. ¿Qué sabemos y qué podemos hacer? Aten Primaria 2003; 31(8):000-0. Disponible en: http:/www.elsevier.es/sites/defaultfiles/elsevier/pdf/27/27v31n08a13047737pdf001.pdt

8. Kivimäki M y col. Work stress and risk of cardiovascular mortality :prospective cohort study of industrial employees. BMJ 2002;325:857.

9. Sidelski D y Bergman G. El estrés laboral se asoció a mayor riesgo de mortalidad cardiovascular en trabajadores de la industria. Evid Act Pract Ambul Jul-Ago 2003;6(4):113. Comentado de: Kivimäki M y col. Work stress and risk of cardiovascular mortality :prospective cohort study of industrial employees. BMJ 2002;325:857. Disponible en castellano: http://Mww.foroaps.orgfiles/laboral.pdf

10. De Vogli R y col. Negative aspects of close relationships are associated with and increased risk of heart cisease Arch Intem Med. 2007:167 (18):1951-1957.

11. Lee S y col. Caregiving and risk of coronary heart disease in U.S. women: a prospective study. Am J Prev Med 2003;24:113-9.

12. Freedman y col. Mortality associated with caregiving, general stress, and caregiving-related stress in elderly women: results of caregiver-study of osteoporotic fractures. $J$ Am Geriatr Soc. 2010 May:58(5):937-43. Epub 2010 Mar 30

13. Schulz R y col. Caregiving as a risk factor for mortality: the Caregiver Health Effects Study. JAMA 1999:282:2215-9.

14. Yususf S y col. Effect of potentially modifiable riskfactors associated with myocardial infarction in 52 countries (the INTERHEART study) Lancet 2004:364:937-52

14. Yusust S y col. Effect of potentially modifiable riskfactors associated with myocardial infarction in 52 counties (the INTERHEART study) Lancet 2004;364:937-52.
15. Gulliksson M y col. Randomized Controlled Trial of Cognitive Behavioral Therapy vs. Standard Treatment to Prevent Recurrent Cardiovascular Events in Patients With Coronary Heart Disease, 15. Gulliksson M y col. Randomized Controlled Trial of Cognitive Behavioral Therapy vs. Standard Treatment to
Secondary Prevention in Uppsala Primary Health Care Project (SUPRIM).Arch Intem Med 2011;171(2):134-140.

16. Whalley B y col. Psychological interventions for coronary heart disease. Cochrane Database Syst Rev. 2011 Aug 10;(8):CD002902.

* Servicio de Nefrología del Hospital Italiano de Buenos Aires carlos.musso@ hospitalitaliano.org.ar 